\title{
An Investigation into Properties of Jackknifed and Bootstrapped Liu-type Estimator*
}

\author{
Yogendra P. Chaubey \\ Concordia University, Montréal, Canada \\ Shalini CHANDRA \\ Banasthali University, Rajasthan, India \\ Mansi Khurana \\ The North Cap University Gurgaon, India
}

\begin{abstract}
In 2003, Liu [16] proposed a new estimator dealing with the problem of multicollinearity in linear regression model pointing out a drawback of ridge estimator used in this context. This new estimator, called Liu-type estimator was demonstrated to have lesser mean squared error than ridge estimator and ordinary least squares estimator, however, it may carry a large amount of bias. In the present paper, we propose different estimators in order to reduce the bias of Liu-type estimator, one using the Jackknife technique and other using the technique proposed in Kadiyala [11]. We also investigate the Bootstrap method of bias correction on the Liu-type estimator as well. The bias and mean squared error of these estimators have been compared using a simulation study as well as a numerical example.
\end{abstract}

Key Words: Multicollinearity, Liu-type estimator, Ridge estimator, Jackknife technique, Bootstrap technique.

\section{Introduction}

In 2003, Liu [16] pointed out that the ordinary ridge estimator (ORE) given by Hoerl and Kennard [8] may still be ill-conditioned if $k$, the biasing constant, is chosen to be very small and to overcome this problem, proposed a new estimator called the Liu-type estimator

*This research is partly based on doctoral thesis of Mansi Khurana, completed at Banasthali University, Rajasthan, India 
(LTE). This estimator should be differentiated from an earlier proposal by Liu [15] in 1993 that has been called as Liu estimator by the researchers [see Alheety and Kibria [3]]. The basic difference between the two estimators is that the former is based on two constants whereas the latter requires one constant. Thus the Liu-type estimator may be considered an improvement over the Liu estimator. It may be noted that both of these may suffer serious bias based on the values of the biasing parameter(s).

In order to reduce the bias of the Liu estimator, Akdeniz and Kaçiranlar [1] proposed an almost unbiased Liu estimator following Kadiyala [11] and Ohtani [18]. They compared the performance of almost unbiased Liu estimator with the Liu estimator and OLSE in terms of mean squared error (MSE). Recently, following the lines of Singh et. al. [19], Akdeniz and Akdeniz [5] performed jackknife technique on the Liu estimator and, by ignoring some terms, obtained the form that is the same as the almost unbiased Liu estimator given by Akdeniz

and Kaçiranlar [1]. They also proposed a new estimator by incorporating Liu estimator in jackknifed Liu estimator and compared its performance with the Liu and jackknifed Liu estimator. Alheety and Kibria [3] extended Liu and almost unbiased Liu estimators for the regression model with correlated errors and multicollinearity. Other references around Liu estimator include Kaciranlar et. al. [10], Kibria [14] etc..

In the present paper, our aim is to improve upon the bias of LTE using jackknife and bootstrap methods and to investigate their performance in comparison to other estimators of interest. The form of the jackknifed LTE may also be used to write down the exact jackknifed Liu estimator in contrast to the one in Akdeniz and Akdeniz [5]. Section 2 consists of the model and the estimators; we propose two new estimators namely jackknife Liu-type estimator (JLTE) and almost unbiased Liu-type estimator (AULTE). Section 3.1 gives the comparison between the bias of LTE and JLTE, where as Section 3.2 compares the MSE's of LTE and AULTE. Section 4 and Section 5 consist of the simulation study and numerical illustration respectively and Section 6 gives some concluding remarks.

\section{The Model and the Estimators}

Consider the following linear regression model

$$
y=X \beta+u,
$$

where $X$ is an $n \times p$ matrix with full column rank i.e. $p, y$ is an $n \times 1$ response vector, $\beta$ is a $p \times 1$ vector of regression coefficients and $u$ is an $n \times 1$ vector of disturbances with $E(u)=0$ and $E\left(u u^{\prime}\right)=\sigma^{2} I$. 
The OLSE for $\beta$ in model (2.1) is

$$
\hat{\beta}_{O L S E}=\left(X^{\prime} X\right)^{-1} X^{\prime} y
$$

where as the commonly used biased estimator in the presence of multicollinearity among independent variables is the ORE, given by

$$
\hat{\beta}_{\text {ORE }}=\left(X^{\prime} X+k I\right)^{-1} X^{\prime} y, k \geq 0 .
$$

Liu [16] drew the attention to one of the drawbacks of ORE that $k$ chosen in ORE should be small and the small $k$ may not be large enough to deal with the ill-conditioning problem and $\left(X^{\prime} X+k I\right)$ may still be ill-conditioned. To overcome this problem, a new estimator called the LTE was proposed which is given by

$$
\hat{\beta}_{L T E}=\left(X^{\prime} X+k I\right)^{-1}\left(X^{\prime} y-d \hat{\beta}\right),
$$

where $k>0,-\infty<d<\infty$ and $\hat{\beta}$ can be any estimator of $\beta$ but in our study, we take it to be $\hat{\beta}_{O L S E}$. Hence, the resulting estimator becomes

$$
\hat{\beta}_{L T E}=\left(X^{\prime} X+k I\right)^{-1}\left(X^{\prime} y-d \hat{\beta}_{O L S E}\right) .
$$

The form of Liu estimator may be obtained from the above by substituting 1 or $k$ and $d$ for $-d$. It was shown that under certain parametric conditions, LTE has lesser MSE than that of ORE. A method to select the optimal values of $k$ and $d$ was also proposed. Like ORE, LTE also carries a substantial amount of bias and in order to reduce the bias of LTE, we perform jackknife technique on this estimator following Singh et. al. [19] in the next subsection.

\subsection{Jackknife Liu-type Estimator}

First let us transform the model in (2.1) as

$$
y=Z \gamma+u,
$$

where, $Z=X G$ and $\gamma=G^{\prime} \beta$. Here $G$ is a $p \times p$ matrix whose columns are normalized eigen vectors of $X^{\prime} X$. Also, $Z^{\prime} Z=G^{\prime} X^{\prime} X G=\Lambda=\operatorname{diag}\left(\lambda_{1}, \ldots, \lambda_{p}\right), \lambda_{i}$ being the $i^{\text {th }}$ eigenvalue of $X^{\prime} X$. The ORE of $\gamma$ may be written as

$$
\hat{\gamma}_{O R E}=(\Lambda+k I)^{-1} Z^{\prime} y=A^{-1} Z^{\prime} y=A^{-1} \Lambda \hat{\gamma}_{O L S E}=\left(I-A^{-1} k I\right) \hat{\gamma}_{O L S E},
$$

where $k \geq 0, A=\Lambda+k I$. Also, LTE of $\gamma$ is written as

$$
\hat{\gamma}_{L T E}=(\Lambda+k I)^{-1}\left(Z^{\prime} y-d \hat{\gamma}_{O L S E}\right)=A^{-1}(\Lambda-d I) \hat{\gamma}_{O L S E}=\left[I-A^{-1}(k I+d I)\right] \hat{\gamma}_{O L S E} .
$$


The above estimator may also be written as

$$
\begin{aligned}
\hat{\gamma}_{L T E} & =A^{-1} Z^{\prime} y-A^{-1} d \hat{\gamma}_{O L S E} \\
& =\hat{\gamma}_{O R E}-(\Lambda+k I)^{-1} d \Lambda^{-1} Z^{\prime} y \\
& =\hat{\gamma}_{O R E}-\alpha,
\end{aligned}
$$

where $\alpha=(\Lambda+k I)^{-1} d \Lambda^{-1} Z^{\prime} y$. Now, performing jackknife technique on LTE, we first obtain $\hat{\gamma}_{L T E(-i)}$, the estimator after deleting the $i^{\text {th }}$ observation in data as given by,

$$
\hat{\gamma}_{L T E(-i)}=\hat{\gamma}_{O R E(-i)}-\alpha_{(-i)} .
$$

We already know the value of $\hat{\gamma}_{O R E(-i)}$ (see Singh et. al. [19] and Khurana et. al. [12]), so we are left with obtaining $\alpha_{(-i)}$. We have,

$$
\alpha=\left(Z^{\prime} Z+k I\right)^{-1} d\left(Z^{\prime} Z\right)^{-1} Z^{\prime} y .
$$

So,

$$
\begin{aligned}
\alpha_{(-i)} & =\left(Z^{\prime} Z-z_{i} z_{i}^{\prime}+k I\right)^{-1} d\left(Z^{\prime} Z-z_{i} z_{i}^{\prime}\right)^{-1}\left(Z^{\prime} y-z_{i} y_{i}\right) \\
& =\left(A-z_{i} z_{i}^{\prime}\right)^{-1} d\left(\Lambda-z_{i} z_{i}^{\prime}\right)^{-1}\left(Z^{\prime} y-z_{i} y_{i}\right) \\
& =\left[A^{-1}+\frac{A^{-1} z_{i} z_{i}^{\prime} A^{-1}}{1-w_{i}}\right] d\left[\Lambda^{-1}+\frac{\Lambda^{-1} z_{i} z_{i}^{\prime} \Lambda^{-1}}{1-u_{i}}\right]\left[Z^{\prime} y-z_{i} y_{i}\right],
\end{aligned}
$$

where $A=\left(Z^{\prime} Z+k I\right), w_{i}=z_{i}^{\prime} A^{-1} z_{i}$ and $u_{i}=z_{i}^{\prime} \Lambda^{-1} z_{i}$. The above equation becomes

$$
\begin{aligned}
\alpha_{(-i)}= & {\left[A^{-1} d+\frac{A^{-1} z_{i} z_{i}^{\prime} A^{-1} d}{\left(1-w_{i}\right)}\right]\left[\Lambda^{-1} Z^{\prime} y+\frac{\Lambda^{-1} z_{i} z_{i}^{\prime} \Lambda^{-1} Z^{\prime} y}{\left(1-u_{i}\right)}-\Lambda^{-1} z_{i} y_{i}-\frac{\Lambda^{-1} z_{i} z_{i}^{\prime} \Lambda^{-1} z_{i} y_{i}}{\left(1-u_{i}\right)}\right] } \\
= & A^{-1} d \Lambda^{-1} Z^{\prime} y+\frac{A^{-1} d \Lambda^{-1} z_{i} z_{i}^{\prime} \Lambda^{-1} Z^{\prime} y}{\left(1-u_{i}\right)}-A^{-1} d \Lambda^{-1} z_{i} y_{i}-\frac{A^{-1} d \Lambda^{-1} z_{i} z_{i}^{\prime} \Lambda^{-1} z_{i} y_{i}}{\left(1-u_{i}\right)} \\
& +\frac{A^{-1} z_{i} z_{i}^{\prime} A^{-1} d \Lambda^{-1} Z^{\prime} y}{\left(1-w_{i}\right)}+\frac{A^{-1} z_{i} z_{i}^{\prime} A^{-1} d \Lambda^{-1} z_{i} z_{i}^{\prime} \Lambda^{-1} Z^{\prime} y}{\left(1-w_{i}\right)\left(1-u_{i}\right)}-\frac{A^{-1} z_{i} z_{i}^{\prime} A^{-1} d \Lambda^{-1} z_{i} y_{i}}{\left(1-w_{i}\right)} \\
& -\frac{A^{-1} z_{i} z_{i}^{\prime} A^{-1} d \Lambda^{-1} z_{i} z_{i}^{\prime} \Lambda^{-1} z_{i} y_{i}}{\left(1-w_{i}\right)\left(1-u_{i}\right)} .
\end{aligned}
$$

The above equation simplifies to

$$
\begin{aligned}
\alpha_{(-i)}= & \alpha+\frac{A^{-1} d \Lambda^{-1} z_{i} z_{i}^{\prime} \hat{\gamma}_{O L S E}}{\left(1-u_{i}\right)}-A^{-1} d \Lambda^{-1} z_{i} y_{i}-\frac{A^{-1} d \Lambda^{-1} z_{i} u_{i} y_{i}}{\left(1-u_{i}\right)} \\
& +\frac{A^{-1} z_{i} z_{i}^{\prime} A^{-1} d \hat{\gamma}_{O L S E}}{\left(1-w_{i}\right)}+\frac{A^{-1} z_{i} v_{i} z_{i}^{\prime} \hat{\gamma}_{O L S E}}{\left(1-w_{i}\right)\left(1-u_{i}\right)}-\frac{A^{-1} z_{i} v_{i} y_{i}}{\left(1-w_{i}\right)}-\frac{A^{-1} z_{i} v_{i} u_{i} y_{i}}{\left(1-u_{i}\right)\left(1-w_{i}\right)} \\
\alpha_{(-i)}= & \alpha-\frac{A^{-1} d \Lambda^{-1} z_{i} e_{i}}{\left(1-u_{i}\right)}+\frac{A^{-1} z_{i} z_{i}^{\prime} \alpha}{\left(1-w_{i}\right)}-\frac{A^{-1} z_{i} v_{i} e_{i}}{\left(1-w_{i}\right)\left(1-u_{i}\right)}
\end{aligned}
$$


where $e_{i}=y_{i}-z_{i}^{\prime} \hat{\gamma}_{O L S E}$, the OLSE residuals and $v_{i}=z_{i}^{\prime} A^{-1} d \Lambda^{-1} z_{i}$. For the following equation, please refer to Khurana et al.[12],

$$
\hat{\gamma}_{O R E(-i)}=\hat{\gamma}_{O R E}-\frac{A^{-1} z_{i} e_{i R}}{\left(1-w_{i}\right)}
$$

where $e_{i R}=y_{i}-z_{i}^{\prime} \hat{\gamma}_{O R E}$, the residuals of ORE.

Now, using (2.10) and (2.11) in (2.8), we get

$$
\hat{\gamma}_{L T E(-i)}=\hat{\gamma}_{L T E}-\frac{A^{-1} z_{i} e_{i R}}{\left(1-w_{i}\right)}+\frac{A^{-1} d \Lambda^{-1} z_{i} e_{i}}{\left(1-u_{i}\right)}-\frac{A^{-1} z_{i} z_{i}^{\prime} \alpha}{\left(1-w_{i}\right)}+\frac{A^{-1} z_{i} v_{i} e_{i}}{\left(1-w_{i}\right)\left(1-u_{i}\right)} .
$$

Now,

$$
\hat{\gamma}_{L T E}-\hat{\gamma}_{L T E(-i)}=\frac{A^{-1} z_{i} e_{i R}}{\left(1-w_{i}\right)}-\frac{A^{-1} d \Lambda^{-1} z_{i} e_{i}}{\left(1-u_{i}\right)}+\frac{A^{-1} z_{i} z_{i}^{\prime} \alpha}{\left(1-w_{i}\right)}-\frac{A^{-1} z_{i} v_{i} e_{i}}{\left(1-w_{i}\right)\left(1-u_{i}\right)} .
$$

Defining the pseudo values,

$$
Q_{i}=\hat{\gamma}_{L T E}+n\left(1-w_{i}\right)\left(\hat{\gamma}_{L T E}-\hat{\gamma}_{L T E(-i)}\right) .
$$

Using (2.12) in (2.13), we get the pseudo values as

$$
Q_{i}=\hat{\gamma}_{L T E}+n\left(1-w_{i}\right)\left[\frac{A^{-1} z_{i} e_{i R}}{\left(1-w_{i}\right)}-\frac{A^{-1} d \Lambda^{-1} z_{i} e_{i}}{\left(1-u_{i}\right)}+\frac{A^{-1} z_{i} z_{i}^{\prime} \alpha}{\left(1-w_{i}\right)}-\frac{A^{-1} z_{i} v_{i} e_{i}}{\left(1-w_{i}\right)\left(1-u_{i}\right)}\right] .
$$

Averaging over $Q_{i}$ will give the JLTE as

$$
\begin{aligned}
\bar{Q}= & \sum_{i=1}^{n} Q_{i} / n \\
= & \hat{\gamma}_{L T E}+A^{-1} \sum_{i=1}^{n} z_{i} e_{i R}-A^{-1} d \Lambda^{-1} \sum_{i=1}^{n} z_{i} e_{i} \frac{\left(1-w_{i}\right)}{\left(1-u_{i}\right)}+A^{-1} \sum_{i=1}^{n} z_{i} z_{i}^{\prime} \alpha \\
& -A^{-1} \sum_{i=1}^{n} z_{i} \frac{v_{i}}{\left(1-u_{i}\right)} e_{i} \\
= & \hat{\gamma}_{L T E}+A^{-1} Z^{\prime} e_{R}-A^{-1} d \Lambda^{-1} Z^{\prime} D_{1} e+A^{-1} Z^{\prime} Z \alpha-A^{-1} Z^{\prime} D_{2} e .
\end{aligned}
$$

where $D_{1}=\operatorname{diag}\left\{\left(\frac{1-w_{i}}{1-u_{i}}\right)\right\}, i=1, \ldots, n$ and $D_{2}=\operatorname{diag}\left\{\left(\frac{v_{i}}{1-u_{i}}\right)\right\}, i=1, \ldots, n$. Now, JLTE will be equal to $\bar{Q}$.

$$
\begin{aligned}
\hat{\gamma}_{J L T E} & =\hat{\gamma}_{L T E}+A^{-1} Z^{\prime} e_{R}+A^{-1} \Lambda \alpha-A^{-1} d \Lambda^{-1} Z^{\prime} D_{1} e-A^{-1} Z^{\prime} D_{2} e \\
& =\hat{\gamma}_{O R E}-\alpha+A^{-1} Z^{\prime} y-A^{-1} \Lambda \hat{\gamma}_{O R E}+A^{-1} \Lambda \alpha-A^{-1} d \Lambda^{-1} Z^{\prime} D_{1} e-A^{-1} Z^{\prime} D_{2} e \\
& =\left(I-A^{-1} \Lambda\right) \hat{\gamma}_{O R E}-\left(I-A^{-1} \Lambda\right) \alpha+A^{-1} Z^{\prime} y-A^{-1} d \Lambda^{-1} Z^{\prime} D_{1} e-A^{-1} Z^{\prime} D_{2} e \\
\hat{\gamma}_{J L T E} & =\left(I-A^{-1} \Lambda\right) \hat{\gamma}_{L T E}+\hat{\gamma}_{O R E}-A^{-1} d \Lambda^{-1} Z^{\prime} D_{1} e-A^{-1} Z^{\prime} D_{2} e .
\end{aligned}
$$


We see that the final form of JLTE is not very compact and it will be quite tedious to compare its MSE with LTE. Hence, we propose another estimator called AULTE following Kadiyala [11] in the next subsection.

\subsection{Almost unbiased Liu-type Estimator}

The LTE for $\gamma$ is

$$
\hat{\gamma}_{L T E}=(\Lambda+k I)^{-1}(\Lambda-d I) \hat{\gamma}_{O L S E},
$$

where $k>0$ and $-\infty<d<\infty$. The bias of LTE is given by

$$
\begin{aligned}
\operatorname{Bias}\left(\hat{\gamma}_{L T E}\right) & =E\left(\hat{\gamma}_{L T E}\right)-\gamma \\
& =-\left[I-A^{-1}(\Lambda-d I)\right] \gamma
\end{aligned}
$$

Following Kadiyala [11] and using (2.15) and (2.16), the bias corrected Liu-type estimator (BCLTE) of $\gamma$ is given by

$$
\begin{aligned}
\hat{\gamma}_{B C L T E} & =\hat{\gamma}_{L T E}-\operatorname{Bias}\left(\hat{\gamma}_{L T E}\right) \\
& =\hat{\gamma}_{L T E}+\left[I-A^{-1}(\Lambda-d I)\right] \gamma
\end{aligned}
$$

Now, following Ohtani [18], if we replace $\gamma$ by $\hat{\gamma}_{L T E}$ to make it operational, we get the AULTE as

$$
\begin{aligned}
\hat{\gamma}_{A U L T E} & =\hat{\gamma}_{L T E}+\left[I-A^{-1}(\Lambda-d I)\right] \hat{\gamma}_{L T E} \\
& =\hat{\gamma}_{L T E}+A^{-1}(k I+d I) \hat{\gamma}_{L T E} \\
& =\left[I+A^{-1}(k I+d I)\right] \hat{\gamma}_{L T E} .
\end{aligned}
$$

We know that $\hat{\gamma}_{L T E}=A^{-1}(\Lambda-d I) \hat{\gamma}_{O L S E}=\left[I-A^{-1}(k I+d I)\right] \hat{\gamma}_{O L S E}$. Using this, the above estimator may be written as

$$
\hat{\gamma}_{A U L T E}=\left[I-A^{-2}(k I+d I)^{2}\right] \hat{\gamma}_{O L S E}
$$

The bias of AULTE is given by

$$
\operatorname{Bias}\left(\hat{\gamma}_{A U L T E}\right)=-A^{-2}(k I+d I)^{2} \gamma .
$$

It is easy to show that $\operatorname{Bias}\left(\hat{\gamma}_{A U L T E}\right)<\operatorname{Bias}\left(\hat{\gamma}_{L T E}\right)$ following the proof related to bias given in the next section. And using AULTE, we can easily compare the MSE's of LTE and AULTE. (Theorem 3.1).

In the next section, we compare the biases of LTE and JLTE. 


\section{Comparison between LTE, JLTE and AULTE}

\subsection{Bias Comparison between LTE and JLTE}

From (2.16), we have the bias of LTE as

$$
\begin{aligned}
\operatorname{Bias}\left(\hat{\gamma}_{L T E}\right) & =-\left[I-A^{-1}(\Lambda-d I)\right] \gamma \\
& =-(k I+d I) A^{-1} \gamma .
\end{aligned}
$$

Now, for the bias of JLTE, we have

$$
\begin{aligned}
\hat{\gamma}_{J L T E}=(I- & \left.A^{-1} \Lambda\right) \hat{\gamma}_{L T E}+\hat{\gamma}_{O R E}-A^{-1} d \Lambda^{-1} Z^{\prime} D_{1} e-A^{-1} Z^{\prime} d_{2} e \\
\operatorname{Bias}\left(\hat{\gamma}_{J L T E}\right) & =E\left(\hat{\gamma}_{J L T E}\right)-\gamma \\
& =\left(I-A^{-1} \Lambda\right) E\left(\hat{\gamma}_{L T E}\right)+E\left(\hat{\gamma}_{O R E}\right)-\gamma \\
& =\left(I-A^{-1} \Lambda\right) A^{-1}(\Lambda-d I) \gamma+\left(I-A^{-1} k I\right) \gamma-\gamma \\
& =-\left[I-\left(I-A^{-1} \Lambda\right) A^{-1}(\Lambda-d I)-\left(I-A^{-1} k I\right)\right] \gamma
\end{aligned}
$$

On simplifying (3.2), we get

$$
\operatorname{Bias}\left(\hat{\gamma}_{J L T E}\right)=-k(k I+d I) A^{-2} \gamma
$$

From (3.1) and (3.3), we get

$$
\operatorname{Bias}\left(\hat{\gamma}_{J L T E}\right)=k A^{-1} \operatorname{Bias}\left(\hat{\gamma}_{L T E}\right) .
$$

Comparing the $i^{\text {th }}$ components,

$$
\operatorname{Bias}\left(\hat{\gamma}_{J L T E}\right)_{i}=\frac{k}{k+\lambda_{i}} \operatorname{Bias}\left(\hat{\gamma}_{L T E}\right)_{i},
$$

where $0<\frac{k}{k+\lambda_{i}}<1$. This proves that the $\operatorname{Bias}\left(\hat{\gamma}_{J L T E}\right)_{i}$ is lesser than the $\operatorname{Bias}\left(\hat{\gamma}_{L T E}\right)_{i} \forall i$.

Next we find the expression for the variance of JLTE. JLTE in (2.14) may be written as

$$
\begin{aligned}
\hat{\gamma}_{J L T E}= & \left(I-A^{-1} \Lambda\right) A^{-1}(\Lambda-d I) \hat{\gamma}_{O L S E}+\left(I-A^{-1} k I\right) \hat{\gamma}_{O L S E} \\
& -A^{-1} d \Lambda^{-1} Z^{\prime} D_{1} e-A^{-1} Z^{\prime} D_{2} e \\
= & {\left[\left(I-A^{-1} \Lambda\right) A^{-1}(\Lambda-d I)+\left(I-A^{-1} k I\right)\right] \hat{\gamma}_{O L S E} } \\
& -\left[A^{-1} d \Lambda^{-1} Z^{\prime} D_{1}+A^{-1} Z^{\prime} D_{2}\right] e .
\end{aligned}
$$


Now using the above form and the fact that $\operatorname{cov}\left(\hat{\gamma}_{O L S E}, e\right)=0$, we get

$$
\begin{aligned}
\operatorname{Var}\left(\hat{\gamma}_{J L T E}\right)= & {\left[\left(I-A^{-1} \Lambda\right) A^{-1}(\Lambda-d I)+\left(I-A^{-1} k I\right)\right] V\left(\hat{\gamma}_{O L S E}\right) } \\
& {\left[\left(I-A^{-1} \Lambda\right) A^{-1}(\Lambda-d I)+\left(I-A^{-1} k I\right)\right]^{\prime} } \\
& +\left[A^{-1} d \Lambda^{-1} Z^{\prime} D_{1}+A^{-1} Z^{\prime} D_{2}\right] V(e)\left[A^{-1} d \Lambda^{-1} Z^{\prime} D_{1}+A^{-1} Z^{\prime} D_{2}\right]^{\prime} \\
= & \sigma^{2}\left[\left(I-A^{-1} \Lambda\right) A^{-1}(\Lambda-d I)+\left(I-A^{-1} k I\right)\right] \Lambda^{-1} \\
& {\left[\left(I-A^{-1} \Lambda\right) A^{-1}(\Lambda-d I)+\left(I-A^{-1} k I\right)\right]^{\prime} } \\
& +\sigma^{2}\left[A^{-1} d \Lambda^{-1} Z^{\prime} D_{1}+A^{-1} Z^{\prime} D_{2}\right]\left[I-Z \Lambda^{-1} Z^{\prime}\right]\left[A^{-1} d \Lambda^{-1} Z^{\prime} D_{1}+A^{-1} Z^{\prime} D_{2}\right]^{\prime} .
\end{aligned}
$$

Using (3.2) and (3.5), MSE of JLTE becomes

$$
\begin{aligned}
M S E\left(\hat{\gamma}_{J L T E}\right)= & \operatorname{Var}\left(\hat{\gamma}_{J L T E}\right)+\operatorname{Bias}\left(\hat{\gamma}_{J L T E}\right) \operatorname{Bias}\left(\hat{\gamma}_{J L T E}\right)^{\prime} \\
= & \sigma^{2}\left[\left(I-A^{-1} \Lambda\right) A^{-1}(\Lambda-d I)+\left(I-A^{-1} k I\right)\right] \Lambda^{-1} \\
& {\left[\left(I-A^{-1} \Lambda\right) A^{-1}(\Lambda-d I)+\left(I-A^{-1} k I\right)\right]^{\prime} } \\
& +\sigma^{2}\left[A^{-1} d \Lambda^{-1} Z^{\prime} D_{1}+A^{-1} Z^{\prime} D_{2}\right]\left[I-Z \Lambda^{-1} Z^{\prime}\right]\left[A^{-1} d \Lambda^{-1} Z^{\prime} D_{1}+A^{-1} Z^{\prime} D_{2}\right]^{\prime} \\
& +\left[I-\left(I-A^{-1} \Lambda\right) A^{-1}(\Lambda-d I)-\left(I-A^{-1} k I\right)\right] \gamma \gamma^{\prime} \\
& {\left[I-\left(I-A^{-1} \Lambda\right) A^{-1}(\Lambda-d I)-\left(I-A^{-1} k I\right)\right]^{\prime} . }
\end{aligned}
$$

The variance of LTE is given by

$$
\begin{aligned}
\operatorname{Var}\left(\hat{\gamma}_{L T E}\right) & =\left[A^{-1}(\Lambda-d I)\right] V(\hat{\gamma})\left[A^{-1}(\Lambda-d I)\right]^{\prime} \\
& =\sigma^{2}\left[A^{-1}(\Lambda-d I)\right] \Lambda^{-1}\left[A^{-1}(\Lambda-d I)\right]^{\prime} .
\end{aligned}
$$

Now, using (3.7) and (3.1), we have the MSE of LTE as

$$
\begin{aligned}
\operatorname{MSE}\left(\hat{\gamma}_{L T E}\right)= & \operatorname{Var}\left(\hat{\gamma}_{L T E}\right)+\operatorname{Bias}\left(\hat{\gamma}_{L T E}\right) \operatorname{Bias}\left(\hat{\gamma}_{L T E}\right)^{\prime} \\
= & \sigma^{2} A^{-1}(\Lambda-d I) \Lambda^{-1}\left(A^{-1}(\Lambda-d I)\right)^{\prime} \\
& +\left[I-A^{-1}(\Lambda-d I)\right] \gamma \gamma^{\prime}\left[I-A^{-1}(\Lambda-d I)\right]^{\prime} .
\end{aligned}
$$

Finding the dominance condition of $M S E\left(\hat{\gamma}_{J L T E}\right)$ over $M S E\left(\hat{\gamma}_{L T E}\right)$ is quite complicated, so we compare the SMSE (scalar MSE; trace of the MSE matrix) of the two estimators through a simulation study and a numerical example. In the next subsection, we compare the MSE's of AULTE and LTE.

\subsection{MSE comparison between LTE and AULTE}

Lemma: [Farebrother (1976)] Let $A$ be a positive definite matrix, $\gamma$ be a $p \times 1$ vector. Then $A-\gamma \gamma^{\prime}$ is a nonnegative definite matrix if and only if $\gamma^{\prime} A^{-1} \gamma \leq 1$ is satisfied. 
Theorem 3.1. Let $k>0$ and $-\infty<d<\infty$. Then,

$$
\Delta=\operatorname{MSE}\left(\hat{\gamma}_{A U L T E}\right)-M S E\left(\hat{\gamma}_{L T E}\right),
$$

is a positive definite matrix if and only if the following inequality is satisfied

$$
\gamma^{\prime}\left\{L^{-1}\left[\sigma^{2} H+A^{-4}(k I+d I)^{4} \gamma \gamma^{\prime}\right] L^{-1}\right\}^{-1} \gamma \leq 1,
$$

where $L=A^{-1}(k I+d I)$ and $H=\left[I-A^{-1}(k I+d I)\right]^{2} \Lambda^{-1}\left\{\left[I+A^{-1}(k I+d I)\right]^{2}-I\right\}$.

Proof. Using the fact that $\left(I-A^{-1}(\Lambda-d I)\right)=A^{-1}(k I+d I)$, we can write (3.8) as

$$
M S E\left(\hat{\gamma}_{L T E}\right)=\sigma^{2}\left[I-A^{-1}(k I+d I)\right]^{2} \Lambda^{-1}+A^{-2}(k I+d I)^{2} \gamma \gamma^{\prime} .
$$

Also, the variance of AULTE is given by

$$
\begin{aligned}
\operatorname{Var}\left(\hat{\gamma}_{A U L T E}\right) & =\left[I-A^{-2}(k I+d I)^{2}\right] V\left(\hat{\gamma}_{O L S E}\right)\left[I-A^{-2}(k I+d I)^{2}\right]^{\prime} \\
& =\sigma^{2}\left[I-A^{-2}(k I+d I)^{2}\right] \Lambda^{-1}\left[I-A^{-2}(k I+d I)^{2}\right]^{\prime} .
\end{aligned}
$$

Now, using (2.19) and (3.10), we get the MSE of AULTE as

$$
\begin{aligned}
\operatorname{MSE}\left(\hat{\gamma}_{A U L T E}\right) & =\operatorname{Var}\left(\hat{\gamma}_{A U L T E}\right)+\operatorname{Bias}\left(\hat{\gamma}_{A U L T E}\right) \operatorname{Bias}\left(\hat{\gamma}_{A U L T E}\right)^{\prime} \\
& =\sigma^{2}\left[I-A^{-2}(k I+d I)^{2}\right]^{2} \Lambda^{-1}+A^{-4}(k I+d I)^{4} \gamma \gamma^{\prime} .
\end{aligned}
$$

From (3.9) and (3.11), we get

$$
\begin{aligned}
\Delta & =\sigma^{2}\left[I-A^{-2}(k I+d I)^{2}\right]^{2} \Lambda^{-1}+A^{-4}(k I+d I)^{4} \gamma \gamma^{\prime}-\sigma^{2}\left[I-A^{-1}(k I+d I)\right]^{2} \Lambda^{-1} \\
& -A^{-2}(k I+d I)^{2} \gamma \gamma^{\prime} \\
& =\sigma^{2}\left\{\left[I-A^{-2}(k I+d I)^{2}\right]^{2} \Lambda^{-1}-\left[I-A^{-1}(k I+d I)\right]^{2} \Lambda^{-1}\right\}+A^{-4}(k I+d I)^{4} \gamma \gamma^{\prime} \\
& -A^{-2}(k I+d I)^{2} \gamma \gamma^{\prime} \\
& =\sigma^{2} H+A^{-4}(k I+d I)^{4} \gamma \gamma^{\prime}-A^{-2}(k I+d I)^{2} \gamma \gamma^{\prime}
\end{aligned}
$$

where

$$
\begin{aligned}
H & =\left\{\left[I-A^{-2}(k I+d I)^{2}\right]^{2} \Lambda^{-1}-\left[I-A^{-1}(k I+d I)\right]^{2} \Lambda^{-1}\right\} \\
& =\left[I-A^{-1}(k I+d I)\right]^{2} \Lambda^{-1}\left\{\left[I+A^{-1}(k I+d I)\right]^{2}-I\right\} .
\end{aligned}
$$

It is easy to see that $H$ is a positive definite matrix for the cases where $(i) k>0, d>0$ and $k>d$, (ii) $k>0, d>0$ and $k<d$ and (iii) $k>0, d<0$ and $|k|>|d|$. It is not positive 
definite for the case where $k>0, d<0$ and $|k|<|d|$.

Now, the difference $\Delta$ is positive definite if and only if $L^{-1} \Delta L^{-1}$ is positive definite. We see that

$$
L^{-1} \Delta L^{-1}=L^{-1}\left[\sigma^{2} H+A^{-4}(k I+d I)^{4} \gamma \gamma^{\prime}\right] L^{-1}-\gamma \gamma^{\prime} .
$$

The matrix $\left[\sigma^{2} H+A^{-4}(k I+d I)^{4} \gamma \gamma^{\prime}\right]$ in the above equation is symmetric positive definite. Therefore using the Lemma defined above, we conclude that $L^{-1} \Delta L^{-1}$ is positive definite if and only if following inequality holds

$$
\gamma^{\prime}\left\{L^{-1}\left[\sigma^{2} H+A^{-4}(k I+d I)^{4} \gamma \gamma^{\prime}\right] L^{-1}\right\}^{-1} \gamma \leq 1
$$

This completes the proof.

With the aim of comparing the bias and SMSE of ORE, LTE, JLTE and AULTE under different settings, we carry out a simulation study for the same.

\section{A Simulation Study}

In the present section, we compare the performance of ORE, LTE, JLTE and AULTE in the sense of bias and SMSE with the help of Monte Carlo experiments. Let the model be $y=X \beta+u$ where $u \sim N(0,1)$. Here $\beta$ is taken as the normalized eigen vector corresponding to the largest eigen value of $X^{\prime} X$. To ensure collinearity factor, the explanatory variables are generated from the following equation

$$
x_{i j}=\left(1-\rho^{2}\right)^{\frac{1}{2}} w_{i j}+\rho w_{i p}, i=1,2 \ldots, n ; j=1,2, \ldots, p .
$$

where $w_{i j}$ are independent standard normal pseudo-random numbers and $\rho^{2}$ is the correlation between the two explanatory variables for $j, j^{\prime}<p$ and $j \neq j^{\prime}$. When $j$ or $j^{\prime}=p$, the correlation will be $\rho$. We have taken $\rho=0.9$ and 0.99 to investigate the effects of different degrees of collinearity with sample sizes $n=20$ and 50. Four different combinations for $(k, d)$ are taken as $(0.8,0.4),(1.5,2.5)(3,-1.5)(2,-3)$.

This simulation study is patterned on that of McDonald and Galarnaeu [17]. For computations, the original model is first decomposed into a canonical form to get the estimator of $\gamma$ and then transformed back to the estimator of $\beta$. For these different choices of $\rho, n$ and $(k, d)$, the experiment is replicated 1500 times. The average absolute bias and average SMSE are computed using the following formula

$$
\operatorname{Bias}\left(\hat{\beta}_{i}\right)=\frac{1}{1500} \sum_{j=1}^{1500}\left|\hat{\beta}_{i j}-\beta_{i}\right| .
$$




$$
\operatorname{SMSE}(\hat{\beta})=\frac{1}{1500} \sum_{j=1}^{1500}\left(\hat{\beta}_{j}-\beta\right)^{\prime}\left(\hat{\beta}_{j}-\beta\right) .
$$

Here, $\hat{\beta}_{i j}$ denotes the estimate of $i^{\text {th }}$ parameter in $j^{\text {th }}$ replication and $\beta_{1}, \beta_{2}, \beta_{3}$ and $\beta_{4}$ are the true parameter values.

Our interest also lies in bootstrapping LTE and obtain the bias and SMSE of bias corrected bootstrap Liu-type estimator (BCLE) and compare it with the jackknifed estimator. Firstly, for the model defined in (2.1), we fit the least squares regression equation for full sample of size $n$ and calculate the standardized residuals $\hat{u}_{i}$. Then, draw an $n$ sized bootstrap sample with replacement $\left(\hat{u}_{1}^{(b)}, \hat{u}_{2}^{(b)}, \ldots, \hat{u}_{n}^{(b)}\right)$ from the residuals $\hat{u}_{i}$ 's giving $1 / n$ probability to each $\hat{u}_{i}$. After this, we obtain the bootstrap $y$ values using the resampled residuals keeping the design matrix fixed as shown below

$$
y^{(b)}=X \hat{\beta}_{O L S E}+\hat{u}^{(b)} .
$$

Now, we get the LTE from the first bootstrap sample as

$$
\hat{\beta}_{L T E}^{(b 1)}=\left(X^{\prime} X+k I\right)^{-1}\left(X^{\prime} y-d \hat{\beta}_{O L S E}\right)^{(b 1)} .
$$

Repeat the above steps $B$ times where $B$ is the number of bootstrap resamples. Based on these, the bootstrap Liu-type estimator (BLE) for $\beta$ is given by

$$
\hat{\beta}_{B L E}=\sum_{r=1}^{B} \hat{\beta}_{L T E}^{(b r)} / B .
$$

where $r=1, \ldots, B$. The estimated bias is given by

$$
\text { Bias }_{\text {est }}=\hat{\beta}_{B L E}-\hat{\beta}_{O L S E}
$$

and the BCLE is given by

$$
\hat{\beta}_{B C L E}=\hat{\beta}_{L T E}-\text { Bias }_{\text {est }} .
$$

The number of bootstrap resamples that are considered is 500. Results of the simulation study are given in Tables 1, 2 and 3. From Tables 1 and 2, we see that BCLE is reducing the bias of LTE in almost all of the cases. JLTE has also reduced the bias of LTE in all the cases considered. Bias of LTE is lesser than the bias of ORE, mostly when $(k, d)=(3,-1.5)$. From Table 3, we note that the SMSE of JLTE is lesser than that of LTE's in cases where $(k, d)$ is $(1.5,2.5)$ and $(2,-3)$. The bias of AULTE is also less than that of LTE's and at some places, it is even less than JLTE's. We can see that when $(k, d)$ is $(2,-3)$, the SMSE of AULTE is less than the SMSE's of both JLTE and LTE. Also, the SMSE of BCLE is lesser than LTE's mostly when $(k, d)$ is $(2,-3)$. In the next section, we illustrate our findings using a numerical example. 
Table 1: Bias comparison of ORE, LTE, JLTE and BCLE when $n=20$

\begin{tabular}{|c|c|c|c|c|c|c|}
\hline$\rho$ & $\overline{(k, d)}$ & ORE & LTE & JLTE & AULTE & BCLE \\
\hline \multirow[t]{12}{*}{0.9} & \multirow[t]{3}{*}{$(0.8,0.4)$} & 00359585 & $\overline{00889876}$ & 0.00441062 & 0.00220381 & 0.0077337 \\
\hline & & 56 & 0.01 & 0.00672986 & 0.00 & 0.00 \\
\hline & & 0.01671221 & 0.01963761 & 0.00882148 & 0.00713168 & 0.01066501 \\
\hline & \multirow[t]{3}{*}{$(1.5,2.5)$} & 0.01038602 & 0.03937934 & 0.00050196 & 0.02016977 & 0.00712672 \\
\hline & & 0.01 & 0.04019962 & 0.00839022 & 0838 & 625 \\
\hline & & 0.02 & 0.044 & 0.006 & 8643 & 0.01081683 \\
\hline & \multirow[t]{3}{*}{$(3,-1.5)$} & 0.02288873 & 0.007 & 0.00278312 & .00540309 & 0.00780756 \\
\hline & & 0.030 & 018 & 0.007 & 2749 & 0.00740127 \\
\hline & & 0.03 & 0.02 & 0.00 & 253 & 0.01068230 \\
\hline & \multirow[t]{3}{*}{$(2,-3)$} & 0.012 & 0.01 & 0.006 & 1364 & 0.00828887 \\
\hline & & 0.022 & 0.002 & 0.00 & 0.0( & 0.00804428 \\
\hline & & 0.02880348 & 0.00189039 & 0.01007549 & 6321 & 0.01055753 \\
\hline \multirow[t]{12}{*}{0.99} & \multirow[t]{3}{*}{$(0.8,0.4)$} & 0.00632873 & 0.02032329 & 0.00003615 & 0.01591076 & 0.02178824 \\
\hline & & 0.018 & $0.01 \varepsilon$ & 0.011 & 0.00 & 0.01829871 \\
\hline & & 0.01273458 & 0.0115 & 0.00252733 & 0.00976492 & 0.01537968 \\
\hline & \multirow[t]{3}{*}{$(1.5,2.5)$} & 0.01486287 & 0.075 & 0.01966620 & 0.12106221 & 0.01805438 \\
\hline & & 4 & 0.0 & 0.00 & 0.0 & 202 \\
\hline & & 0.02 & 0.0 & 0.00 & 381 & 0.01772021 \\
\hline & \multirow[t]{3}{*}{$(3,-1.5)$} & 0.029 & 0.00 & 0.00 & 01 & 0.02347565 \\
\hline & & & 0.02 & 0.01 & & 5137 \\
\hline & & 0.0358 & 0.025 & 0.005 & 542 & 0.01406701 \\
\hline & \multirow[t]{3}{*}{$(2,-3)$} & 0.02011554 & 0.0425 & 0.021 & 27883 & 0.02633305 \\
\hline & & & 0.01 & 0.026 & 312 & 0.02638689 \\
\hline & & 0.025 & 0.0098 & 0.00602516 & 47210 & 0.0122 \\
\hline
\end{tabular}


Table 2: Bias comparison of ORE, LTE, JLTE and BCLE when $n=50$

\begin{tabular}{|c|c|c|c|c|c|c|}
\hline$\rho$ & $(k, d)$ & ORE & LTE & JLTE & AULTE & BCLE \\
\hline \multirow[t]{12}{*}{0.9} & \multirow[t]{3}{*}{$\overline{(0.8,0.4)}$} & 0.00946307 & 0.01022269 & 0.00778286 & 0.00763951 & 0.00777550 \\
\hline & & 0.00304358 & 0.00449567 & 0.00005227 & 0.00003726 & 0.00049357 \\
\hline & & 0.00218047 & 0.00033499 & 0.00696886 & 0.00682497 & 0.00766309 \\
\hline & \multirow[t]{3}{*}{$(1.5,2.5)$} & 0.01098788 & 0.01606125 & 0.00719419 & 0.00532347 & 0.00777283 \\
\hline & & 0.00564471 & 0.01482025 & 0.00023196 & 0.00058286 & 0.00027182 \\
\hline & & 0.00196880 & 0.01726912 & 0.00600010 & 0.00374898 & 0.00748086 \\
\hline & \multirow[t]{3}{*}{$(3,-1.5)$} & 0.01461314 & 0.01127850 & 0.00737560 & 0.00771632 & 0.00778080 \\
\hline & & 0.01127577 & 0.00570758 & 0.00011782 & 0.00010636 & 0.00050346 \\
\hline & & 0.01034083 & 0.00156472 & 0.00649076 & 0.00687354 & 0.00767375 \\
\hline & \multirow[t]{3}{*}{$(2,-3)$} & 0.01215312 & 0.00583922 & 0.00796125 & 0.00780553 & 0.00778074 \\
\hline & & 0.00751821 & 0.00355002 & 0.00031200 & 0.00010716 & 0.00068312 \\
\hline & & 0.00482663 & 0.01323040 & 0.00749057 & 0.00702221 & 0.00782039 \\
\hline \multirow[t]{12}{*}{0.99} & \multirow[t]{3}{*}{$(0.8,0.4)$} & 0.00537287 & 0.00421371 & 0.01009930 & 0.00202196 & 0.02421351 \\
\hline & & 0.00578432 & 0.00889184 & 0.00399562 & 0.00584493 & 0.00048458 \\
\hline & & 0.01192998 & 0.00592146 & 0.00767233 & 0.00088292 & 0.02405705 \\
\hline & \multirow[t]{3}{*}{$(1.5,2.5)$} & 0.00084908 & 0.04317427 & 0.01206160 & 0.07905265 & 0.02455552 \\
\hline & & 0.00866138 & 0.02381486 & 0.00842260 & 0.02094144 & 0.00116302 \\
\hline & & 0.01266971 & 0.00612580 & 0.01479676 & 0.08256044 & 0.02284905 \\
\hline & \multirow[t]{3}{*}{$(3,-1.5)$} & 0.00963288 & 0.00745658 & 0.01428730 & 0.01979262 & 0.02417474 \\
\hline & & 0.01478512 & 0.00717720 & 0.00078137 & 0.00029776 & 0.00121435 \\
\hline & & 0.01787113 & 0.02090907 & 0.01448349 & 0.01943132 & 0.02468448 \\
\hline & \multirow[t]{3}{*}{$(2,-3)$} & 0.00414376 & 0.03889093 & 0.03067854 & 0.02041510 & 0.02392582 \\
\hline & & 0.01069389 & 0.00599301 & 0.00266908 & 0.00031289 & 0.00248065 \\
\hline & & 0.01414485 & 0.02884809 & 0.03099984 & 0.01983303 & 0.02562576 \\
\hline
\end{tabular}


Table 3: SMSE comparison of ORE, LTE, JLTE and BCLE

\begin{tabular}{c|c|c|c|c|c|c|c}
\hline$n$ & $\rho$ & $(k, d)$ & ORE & LTE & JLTE & AULTE & BCLE \\
\hline 20 & 0.9 & $(0.8,0.4)$ & 0.476470 & 0.219188 & 0.694739 & 0.4388244 & 1.472460 \\
& & $(1.5,2.5)$ & 0.284077 & 0.737832 & 0.221457 & 5.369156 & 1.477606 \\
& & $(3,-1.5)$ & 0.147666 & 0.606052 & 0.784080 & 1.096411 & 1.476669 \\
& & $(2,-3)$ & 0.218172 & 2.593648 & 2.228677 & 1.163111 & 1.497701 \\
\cline { 2 - 8 } & 0.99 & $(0.8,0.4)$ & 0.373850 & 2.293556 & 1.619370 & 13.00634 & 17.274870 \\
& & $(1.5,2.5)$ & 0.154178 & 39.012490 & 33.334130 & 485.3489 & 17.646990 \\
& & $(3,-1.5)$ & 0.060653 & 4.635836 & 5.009879 & 10.15625 & 17.283510 \\
& & $(2,-3)$ & 0.102604 & 37.548220 & 37.059220 & 10.34917 & 17.561200 \\
\hline 50 & 0.9 & $(0.8,0.4)$ & 0.289831 & 0.247422 & 0.364261 & 0.3540111 & 0.390741 \\
& & $(1.5,2.5)$ & 0.233586 & 0.069411 & 0.287308 & 0.1594167 & 0.387318 \\
& & $(3,-1.5)$ & 0.158766 & 0.258936 & 0.335352 & 0.3598087 & 0.390932 \\
& & $(2,-3)$ & 0.203291 & 0.499424 & 0.417133 & 0.3703853 & 0.395409 \\
\cline { 2 - 8 } 0.99 & $(0.8,0.4)$ & 0.580607 & 0.064050 & 0.689338 & 0.1941529 & 4.266513 \\
& & $(1.5,2.5)$ & 0.252640 & 4.755504 & 1.687548 & 45.31219 & 4.321887 \\
& & $(3,-1.5)$ & 0.089493 & 1.374429 & 1.672291 & 2.811444 & 4.287226 \\
& & $(2,-3)$ & 0.165724 & 8.428380 & 7.610839 & 2.966738 & 4.394909 \\
\hline
\end{tabular}

\section{$5 \quad$ Numerical Illustration}

In this section, we take up real data to illustrate the theoretical findings. We consider the famous dataset on Portland cement originally due to Woods et al. [21] and since then it has been widely used by many researchers (for instance see Hald (1952), Gorman and Toman (1966), Montgomery and Peck (1982), Özkale (2012)). This data arise from an experimental investigation of the dependence of heat evolved during the setting and hardening of Portland cements of varied composition on the percentages of four compounds in the clinkers from which the cement was produced. The four compounds (explanatory variables) considered are tricalcium aluminate $\left(X_{1}\right)$, tricalcium silicate $\left(X_{2}\right)$, tetracalcium aluminoferrite $\left(X_{3}\right)$ and $\beta$-dicalcium silicate $\left(X_{4}\right)$. The heat evolved will be denoted by $y$ and is measured in calories per gram of the cement. Variables have been standardized before the calculations. The data set is given in Table 4 .

The eigen values of $X^{\prime} X$ are $26.82844842,18.91279284,2.23927379,0.01948495$ which gives the condition number as 37.10634. This indicates the presence of strong multicollinearity among the regressors. We use the optimal value of $d$ as given by Liu [16] 
Table 4: Data set

\begin{tabular}{ccccc}
\hline$X_{1}$ & $X_{2}$ & $X_{3}$ & $X_{4}$ & $y$ \\
\hline 7 & 26 & 6 & 60 & 78.5 \\
1 & 29 & 15 & 52 & 74.3 \\
11 & 56 & 8 & 20 & 104.3 \\
11 & 31 & 8 & 47 & 87.6 \\
7 & 52 & 6 & 33 & 95.9 \\
11 & 55 & 9 & 22 & 109.2 \\
3 & 71 & 17 & 6 & 102.7 \\
1 & 31 & 22 & 44 & 72.5 \\
2 & 54 & 18 & 22 & 93.1 \\
21 & 47 & 4 & 26 & 115.9 \\
1 & 40 & 23 & 34 & 83.8 \\
11 & 66 & 9 & 12 & 113.3 \\
10 & 68 & 8 & 12 & 109.4 \\
\hline
\end{tabular}

$$
d_{o p t}=\frac{\sum_{i=1}^{p}\left(\left(\sigma^{2}-k \gamma_{i}^{2}\right) /\left(\lambda_{i}+k\right)^{2}\right)}{\sum_{i=1}^{p}\left(\left(\lambda_{i} \gamma_{i}^{2}+\sigma^{2}\right) / \lambda_{i}\left(\lambda_{i}+k\right)^{2}\right)} .
$$

It was suggested to choose $k$ so that the condition number of $X^{\prime} X+k I$ is reduced to 10 as

$$
\hat{k}=\frac{\lambda_{1}-100 * \lambda_{p}}{99} .
$$

Using the above expressions, we get $\hat{k}=0.2513127$ and $d_{\text {opt }}=-0.01056076$. These values are used to get the estimates of LTE and JLTE which are given in Table 5. It also gives the values of Abias (sum of absolute biases of individual coefficients) and SMSE. SMSE requires the knowledge of $\sigma^{2}$ which has been estimated by using $\hat{\sigma}^{2}=\left(y-X \hat{\beta}_{O L S E}\right)^{\prime}\left(y-X \hat{\beta}_{O L S E}\right) /(n-p)$.

To calculate ORE and its properties in Table 5, we make use of the feasible value of $k$ which is obtained by the optimal formula $k=\frac{p \sigma^{2}}{\beta^{\prime} \beta}$ as given by Hoerl et al. [9], so that

$$
\hat{k}=\frac{p \hat{\sigma}^{2}}{\hat{\beta}_{O L S E}^{\prime} \hat{\beta}_{O L S E}} .
$$

where $\hat{\sigma}^{2}$ is as defined earlier. To see the effects of different values of $(k, d)$ on ORE, LTE and JLTE, we plot the bias and SMSE of these estimators against the different values of $d$ for $k=0.8,1.5$ and 10. We did the calculations for AULTE for different $(k, d)$ and observed 
some dramatically high values of bias mostly in cases when $k$ is small and $d$ is high, so we skipped the idea of putting AULTE in the plots.

Table 5: Estimated values of coefficients, Abias and SMSE of ORE, LTE, JLTE and AULTE

\begin{tabular}{ccccccc}
\hline Estimators & $\beta_{1}$ & $\beta_{2}$ & $\beta_{3}$ & $\beta_{4}$ & Abias & SMSE \\
\hline$\hat{\beta}_{\text {ORE }}$ & 0.517342 & 0.320572 & -0.052067 & -0.377593 & 0.609064 & 0.172062 \\
$\hat{\beta}_{\text {LTE }}$ & 0.500856 & 0.312176 & -0.065651 & -0.384781 & 0.654720 & 0.145385 \\
$\hat{\beta}_{\text {JLTE }}$ & 0.531458 & 0.334313 & -0.039538 & -0.364412 & 0.587632 & 0.152082 \\
$\hat{\beta}_{\text {AULTE }}$ & 0.530644 & 0.331735 & -0.040734 & -0.367263 & 0.562938 & 0.065397 \\
\hline
\end{tabular}

From Figures 5, 3 and 5, we see that the bias of JLTE is always less than the bias of LTE. Also, we can make out the range where the bias of LTE is lesser than the bias of ORE and the point where they are equal. From Figures 2 and 4, we can make out the points where the SMSE of JLTE and LTE becomes little lesser than the SMSE of ORE and the point of intersection of the SMSE's of ORE, LTE and JLTE. Also, from Figure 6 when $k=10$, we see that the SMSE of JLTE and LTE becomes less than that of ORE's in a certain range and the SMSE of JLTE is less than that of LTE's over the range considered.

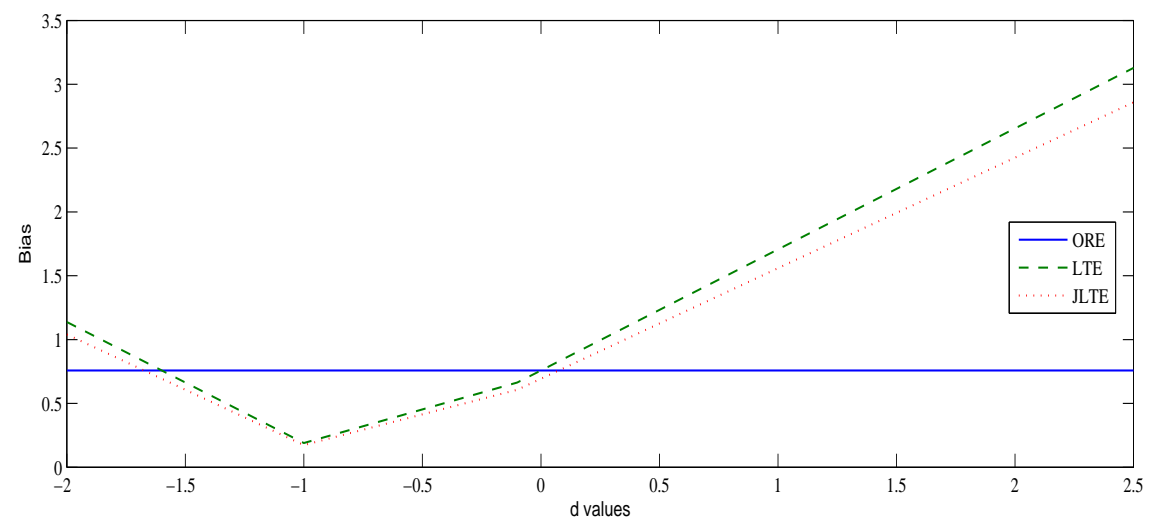

Figure 1: Bias comparison of ORE, LTE and JLTE when $k=0.8$ 


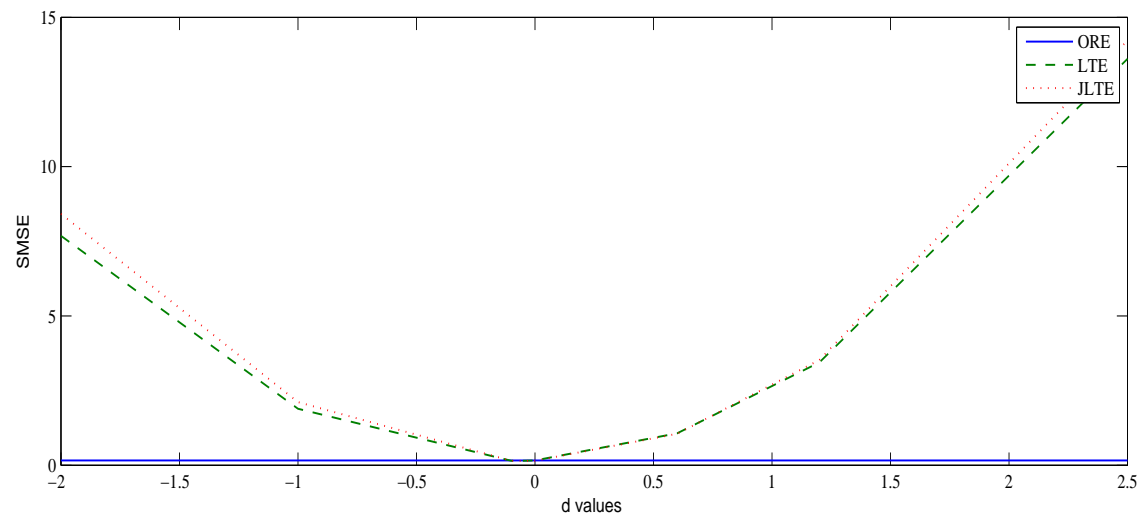

Figure 2: SMSE comparison of ORE, LTE and JLTE when $k=0.8$

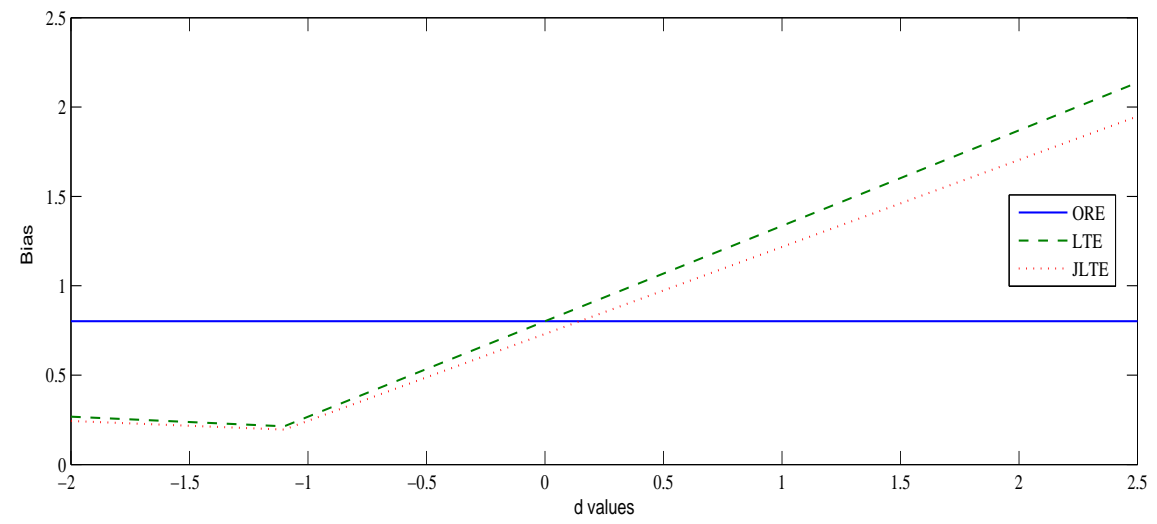

Figure 3: Bias comparison of ORE, LTE and JLTE when $k=1.5$ 


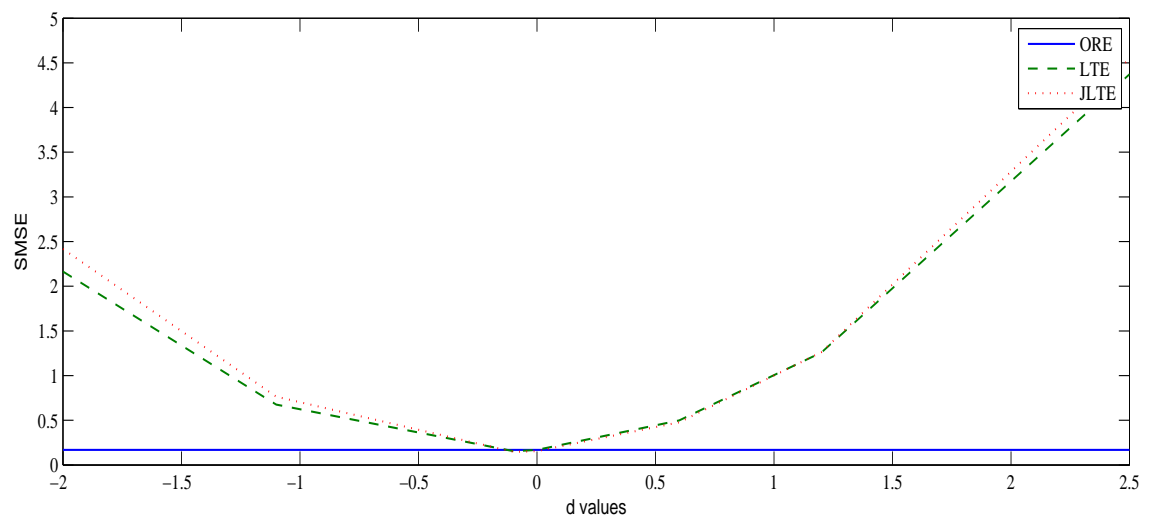

Figure 4: SMSE comparison of ORE, LTE and JLTE when $k=1.5$

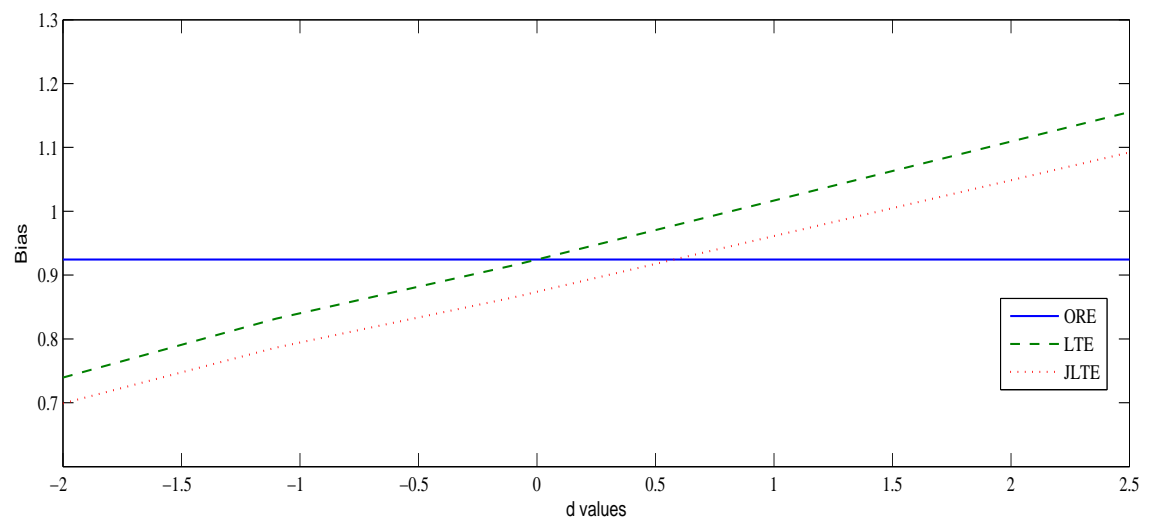

Figure 5: Bias comparison of ORE, LTE and JLTE when $k=10$ 


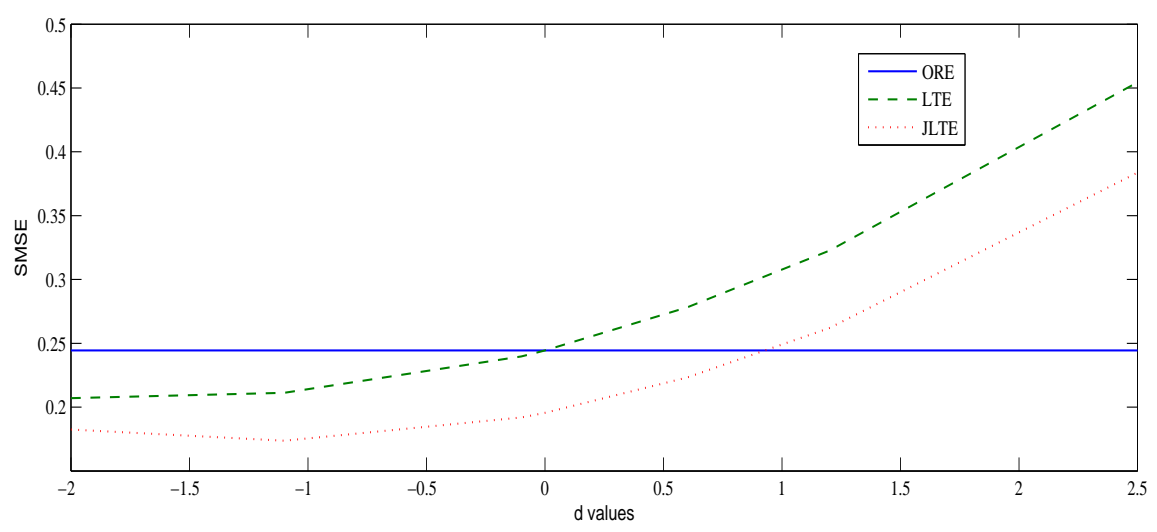

Figure 6: SMSE comparison of ORE, LTE and JLTE when $k=10$

\section{Concluding Remarks}

In the present paper, we performed the jackknifed technique on LTE and obtained the jackknifed estimator, JLTE. We also proposed another almost unbiased Liu type estimator, AULTE. Comparing the bias and SMSE's of ORE, LTE, AULTE and JLTE, we found that, JLTE reduces the bias of LTE and it is also efficient in few cases. AULTE also has a reduced bias than LTE; in some cases, its even lesser than JLTE's and its performance is better in sense of MSE as well in few cases. A Simulation study and a numerical example were shown to support the results. We also performed bootstrap on LTE and found that BCLE's bias is lesser than that of LTE's and it is also efficient in some of the cases.

\section{Acknowledgements}

Y.P. Chaubey would like to acknowledge Natural Sciences and Engineering Research Council of Canada for the partial support of this research through a Discovery Research Grant.

\section{References}

[1] Akdeniz, F. \& Kaçiranlar, S. (1995). On the almost unbiased generalized Liu estimator and unbiased estimation of the bias and MSE. Communications in Statistics-Theory and Methods, 24(7), 1789-1797.

[2] Akdeniz, F. \& Kaçiranlar, S. (2001). More on the new biased estimator in linear regression. Sankhya: The Indian Journal of Statistics, Series B, 63, 321-325. 
[3] Alheety, M. I. \& Kibria, B.M.G. (2009). On the Liu and almost unbiased Liu estimators in the presence of multicollinearity with heteroscedastic or correlated errors. Surveys in Mathematics and its Applications, 4, 155-167.

[4] Alheety, M. I. \& Kibria, B. M. G. (2012). Modified Liu-Type estimator based on $r-k$ class estimator. Communications in Statistics-Theory and Methods, 42(2), 304-319.

[5] Duran, E. A. \& Akdeniz, F. (2012). Efficiency of the modified jackknifed Liu-type estimator. Statistical Papers, 53(2), 265-280.

[6] Farebrother, R. W. (1972). Principal component estimators and minimum mean square error criteria in regression analysis. The Review of Economics and Statistics, 54(3), 332-336.

[7] Hald, A. (1952). Statistical Theory with Engineering Applications. Wiley, New York.

[8] Hoerl, A. E. \& Kennard, R. W. (1970). Ridge regression: Biased estimation for nonorthogonal problems. Technometrics, 12(1), 55-67.

[9] Hoerl, A.E., Kennard, R.W. \& Baldwin, K. (1975). Ridge regression: Some simulations. Communications in Statistics-Theory and Methods, 4, 105-123.

[10] Kaçiranlar, S., Sakallioğlu, S., Akdeniz, F., Styan, G. P. \& Werner, H. J. (1999). A new biased estimator in linear regression and a detailed analysis of the widely-analysed dataset on Portland cement. Sankhya: The Indian Journal of Statistics, Series B, 61, 443-459.

[11] Kadiyala, K. (1984). A class of almost unbiased and efficient estimators of regression coefficients. Economics Letters, 16(3), 293-296.

[12] Khurana, M., Chaubey, Y. P., Chandra, S. (2014). Jackknifing the ridge regression estimator: A revisit. Communications in Statistics-Theory and Methods, 43:24, 52495262

[13] Kibria, B. M. G. (2003). Performance of some new ridge regression estimators. Communications in Statistics-Simulation and Computation, 32(2), 419-435.

[14] Kibria, B.M.G. (2012). Kibria, B. G. (2012). Some Liu and ridge-type estimators and their properties under the ill-conditioned Gaussian linear regression model. Journal of Statistical Computation and Simulation, 82(1), 1-17.

[15] Liu, K. (1993). A new class of biased estimate in linear regression. Communications in Statistics-Theory and Methods, 22(2), 393-402. 
[16] Liu, K. (2003). Using Liu-type estimator to combat collinearity. Communications in Statistics-Theory and Methods, 32(5), 1009-1020.

[17] McDonald, G. C. \& Galarneau, D. I. (1975). A Monte-Carlo evaluation of some Ridgetype estimators. Journal of the American Statistical Association, 70, 407-416.

[18] Ohtani, K. (1986). On small sample properties of the almost unbiased generalized ridge estimator. Communications in Statistics-Theory and Methods, 15(5), 1571-1578.

[19] Singh, B., Chaubey, Y. P. \& Dwivedi, T. D. (1986). An almost unbiased ridge estimator. Sānkhya: The Indian Journal of Statistics, Series B, 48, 342-346.

[20] Stein, C. (1956). Inadmissibility of the usual estimator for the mean of a multivariate normal distribution. In Proceedings of the Third Berkeley symposium on mathematical statistics and probability, 1, 197-206.

[21] Woods, H., Steinour, H. H., and Starke, H. R. (1932). Effect of composition of Portland cement on heat evolved during hardening. Industrial and Engineering Chemistry, 24, 1207-1214. 